\title{
Vibration Characteristics of Piezoelectric Microbeams Based on the Modified Couple Stress Theory
}

\author{
R. Ansari, M. A. Ashrafi, and S. Hosseinzadeh \\ Department of Mechanical Engineering, University of Guilan, P.O. Box 3756, Rasht, Iran \\ Correspondence should be addressed to R. Ansari; r_ansari@guilan.ac.ir
}

Received 25 December 2013; Revised 15 April 2014; Accepted 16 April 2014; Published 7 May 2014

Academic Editor: Tony Murmu

Copyright ( 2014 R. Ansari et al. This is an open access article distributed under the Creative Commons Attribution License, which permits unrestricted use, distribution, and reproduction in any medium, provided the original work is properly cited.

\begin{abstract}
The vibration behavior of piezoelectric microbeams is studied on the basis of the modified couple stress theory. The governing equations of motion and boundary conditions for the Euler-Bernoulli and Timoshenko beam models are derived using Hamilton's principle. By the exact solution of the governing equations, an expression for natural frequencies of microbeams with simply supported boundary conditions is obtained. Numerical results for both beam models are presented and the effects of piezoelectricity and length scale parameter are illustrated. It is found that the influences of piezoelectricity and size effects are more prominent when the length of microbeams decreases. A comparison between two beam models also reveals that the Euler-Bernoulli beam model tends to overestimate the natural frequencies of microbeams as compared to its Timoshenko counterpart.
\end{abstract}

\section{Introduction}

Microbeams in sensors, actuators, and micro-/nanoelectromechanical systems are widely used. Examples can be found in vibration shock sensors [1], electrostatically excited microactuators $[2,3]$, resonant testing method, and atomic force microscope (AFM) $[4,5]$. Some researchers experimentally revealed the size-dependent deformation and vibration behavior of microstructures [6-8]. These experiments showed that the consideration of size-dependent behavior is necessary in the analysis of microbeams. The size-dependent behavior of structures at micron/submicron scales cannot be predicted by classical continuum theories. Therefore, sizedependent continuum models were developed based on some nonclassical continuum theories such as strain gradient theories [9-11], nonlocal elasticity theories [12], and couple stress theories [13-15]. The classical couple stress elasticity theory was introduced by some researchers in 1960s. This theory contains two higher-order material length scale parameters in addition to two Lamé constants. Using this theory, the static and dynamic torsions of a circular cylindrical microbar were investigated by Zhou and Li [16]. The resonant frequencies of a microbeam were studied by Kang and Xi [17]. Using the couple stress theory, Asghari et al. [18] studied the size effects in Timoshenko beams.
Due to difficulties in determining the material length scale parameters, a modified couple stress theory with only one additional length scale parameter was introduced by Yang et al. [19]. Using the modified couple stress theory, the static mechanical properties of Euler-Bernoulli beam model were investigated by Park and Gao [20], and they explicated the bending test outcomes of an epoxy polymeric beam. A Timoshenko beam model was introduced by Ma et al. [21] to study the size-dependent static bending and free vibration properties. Based on the couple stress theory, Reddy [22] defined nonlinear size-dependent EulerBernoulli and Timoshenko functionally graded beam models and by using the Navier solution determined the natural frequency, buckling load, and deflection for beams with simply supported boundary conditions. The modified couple stress theory was also used by Kong et al. [23] to obtain the governing equation and boundary and initial conditions of an Euler-Bernoulli microbeam. Asghari et al. [24] presented a nonlinear Timoshenko beam model based on the modified couple stress theory and determined the nonlinear sizedependent static and vibration behavior. The size-dependent behavior of microbeams made of functionally graded material (FGM) using the modified couple stress theory was studied in [25]. Asghari et al. [26] presented a size-dependent formulation for FGM Timoshenko beams on the basis of 
the modified couple stress theory. Xia et al. [27] presented a nonlinear Euler-Bernoulli beam model based on the modified couple stress theory. The nonlinear size-dependent static bending and buckling and free vibration of beams were investigated in their work. Utilizing the modified couple stress Euler-Bernoulli beam theory, Wang et al. [28] analyzed the nonlinear free vibration behavior of microbeams. They concluded that the nonlinear vibration frequency obtained by their model is higher than that predicted by the classical continuum theory.

Piezoelectric components are widely used in various micro- and nanodevices. Recently, the analysis of nanoscale piezoelectric structures has attracted considerable attention because of their increasing applications. An analytical model was presented by Gheshlaghi and Hasheminejad [29] to predict surface effects on the free vibration of piezoelectric nanowires. They derived the governing equation of motion, the exact expressions for the natural frequencies, and the fundamental buckling voltage for simply supported piezoelectric nanowires. With the consideration of surface effects and surface piezoelectricity, Yan and Jiang [30] studied the electromechanical coupling behavior of piezoelectric nanowires using the Euler-Bernoulli beam theory. The effects of surface stresses on the vibration and buckling of piezoelectric nanowires were analyzed by Wang and Feng [31] by using the Euler-Bernoulli beam model. Surface effect on the buckling of piezoelectric nanofilms was investigated by Zhang et al. [32]. Yan and Jiang [33] studied electromechanical response of a curved piezoelectric nanobeam with the consideration of surface effects. K. Wang and B. Wang [34] analyzed the surface effects on the buckling of piezoelectric nanobeams. The nonlinear vibration of piezoelectric nanobeams was studied by $\mathrm{Ke}$ et al. [35] based on the nonlocal theory. Li et al. [36] investigated the postbuckling of piezoelectric nanobeams with surface effects. The effect of electrostatic force on piezoelectric nanobeams was studied by Liang and Shen [37]. Yan and Jiang [38] studied the vibration and buckling behavior of piezoelectric nanobeams with surface effects.

This paper presents the vibrational analysis of piezoelectric microbeams based on the modified couple stress theory. The governing equations of motion and associated boundary conditions for both Euler-Bernoulli and Timoshenko beam models are derived on the basis of Hamilton's principle. For microbeams under simply supported boundary conditions, an exact expression of natural frequencies is presented. The obtained natural frequencies are normalized by using the natural frequency of classical Euler-Bernoulli beam model and then are used to illustrate the influences of piezoelectricity and size effects on the vibrational behavior of piezoelectric microbeams.

\section{Modified Couple Stress Theory}

In this theory, for infinitesimal deformation, the strain energy density of a linear elastic material is described by

$$
\bar{u}=\frac{1}{2}\left(\sigma_{i j} \varepsilon_{i j}+m_{i j} \chi_{i j}\right) \quad(i, j=1,2,3) .
$$

For isotropic cases, the components of (1) are as follows:

$$
\begin{aligned}
\sigma_{i j} & =\lambda \operatorname{tr}(\varepsilon) \delta_{i j}+2 \mu \varepsilon_{i j}, \\
\varepsilon_{i j} & =\frac{1}{2}\left((\nabla u)_{i}+(\nabla u)_{i}^{T}\right), \\
m_{i j} & =\beta \chi_{i j}=2 l^{2} \mu \chi_{i j}, \\
\chi_{i j} & =\frac{1}{2}\left((\nabla \theta)_{i}+(\nabla \theta)_{i}^{T}\right),
\end{aligned}
$$

where $\sigma_{i j}, \varepsilon_{i j}, \chi_{i j}$, and $m_{i j}$ represent the components of the stress tensor, the strain tensor, the curvature tensor, and the deviatoric part of couple stress tensor, respectively. Also, $u$ and $\theta$ denote the displacement vector and the rotation vector, respectively. It should be noted that $\theta=\operatorname{curl}(u) / 2$. In addition, $\lambda$ and $\mu$ denote the Lamé constants, and $l$ is the material length scale parameter. It is recalled that $\lambda=$ $E v /(1+\nu)(1-2 \nu)$ and $=E / 2(1+\nu)$, where $E$ and $\nu$ are Young's modulus and Poisson's ratio, respectively. The parameter $\beta$ is a higher-order modulus and is considered as the rotational modulus that indicates the resistance of the material versus the gradient of its elements rotation. This parameter is relevant to the shear modulus $\mu$ and the length scale parameter $l$ as $\beta=2 l^{2} \mu$.

\section{Derivation of Governing Equations and Boundary Conditions}

For an Euler-Bernoulli beam, the displacement field is expressed as

$$
\begin{aligned}
& u_{x}=-z \frac{\partial w(x, t)}{\partial x} \\
& u_{y}=0 \\
& u_{z}=w(x, t)
\end{aligned}
$$

in which $u_{x}, u_{y}$, and $u_{z}$ denote the displacement along the axes $x, y$, and $z$, respectively, and $w(x, t)$ is the lateral deflection of the beam.

Also, the displacement field of a Timoshenko beam is

$$
\begin{aligned}
& u_{x}=z \psi(x, t), \\
& u_{y}=0, \\
& u_{z}=w(x, t),
\end{aligned}
$$

where $\psi(x, t)$ denotes the rotation angle of the beam crosssection about the $y$-axis.

The nonzero component of the strain tensor for an EulerBernoulli beam is expressed as

$$
\varepsilon_{x x}=-z \frac{\partial^{2} w}{\partial x^{2}}
$$


and, for a Timoshenko beam, the nonzero components of the strain tensor are in the forms

$$
\begin{aligned}
& \varepsilon_{x x}=z \frac{\partial \psi}{\partial x}, \\
& \varepsilon_{x z}=\frac{1}{2}\left(\psi(x, t)+\frac{\partial w}{\partial x}\right) .
\end{aligned}
$$

From $\theta=\operatorname{curl}(u) / 2$, the relations for the Euler-Bernoulli beam are written as

$$
\theta_{y}=-\frac{\partial w}{\partial x} \theta_{x}=\theta_{z}=0
$$

Substituting (10) into (5), the expression for the only nonzero component of the symmetric part of the curvature tensor is obtained as follows:

$$
\chi_{x y}=\chi_{y x}=-\frac{1}{2}\left(\frac{\partial^{2} w}{\partial x^{2}}\right)
$$

And, for the Timoshenko beam, the obtained relations are

$$
\theta_{y}=\frac{1}{2}\left(\psi(x, t)-\frac{\partial w}{\partial x}\right), \quad \theta_{x}=\theta_{z}=0 .
$$

In addition, substitution of (12) into (5) gives the expression for the only nonzero component of the symmetric part of the curvature tensor for this beam model as

$$
\chi_{x y}=\chi_{y x}=\frac{1}{4}\left(\frac{\partial \psi}{\partial x}-\frac{\partial^{2} w}{\partial x^{2}}\right) .
$$

For Euler-Bernoulli beam, substitution of (11) into (4) yields the nonzero component of the deviatoric part of the couple stress tensor in terms of the kinematic parameters as

$$
m_{x y}=-l^{2} \mu\left(\frac{\partial^{2} w}{\partial x^{2}}\right) .
$$

For the Timoshenko beam, (13) can be substituted into (4) to get

$$
m_{x y}=2 l^{2} \mu\left(\frac{1}{4}\left(\frac{\partial \psi}{\partial x}\right)-\frac{1}{4}\left(\frac{\partial^{2} w}{\partial x^{2}}\right)\right) .
$$

The linear constitutive equations for a homogeneous orthotropic piezoelectric material are in the following forms:

$$
\begin{aligned}
\left\{\begin{array}{c}
\sigma_{x} \\
\sigma_{y} \\
\sigma_{z} \\
\tau_{y z} \\
\tau_{z x} \\
\tau_{x y}
\end{array}\right\}= & {\left[\begin{array}{cccccc}
E_{11} & E_{12} & E_{13} & 0 & 0 & 0 \\
E_{12} & E_{22} & E_{23} & 0 & 0 & 0 \\
E_{13} & E_{23} & E_{33} & 0 & 0 & 0 \\
0 & 0 & 0 & E_{44} & 0 & 0 \\
0 & 0 & 0 & 0 & E_{55} & 0 \\
0 & 0 & 0 & 0 & 0 & E_{66}
\end{array}\right]\left\{\begin{array}{l}
\varepsilon_{x x} \\
\varepsilon_{y y} \\
\varepsilon_{z z} \\
\varepsilon_{y z} \\
\varepsilon_{z x} \\
\varepsilon_{x y}
\end{array}\right\} } \\
& -\left[\begin{array}{ccc}
0 & 0 & e_{1} \\
0 & 0 & e_{2} \\
0 & 0 & e_{3} \\
0 & e_{4} & 0 \\
e_{5} & 0 & 0 \\
0 & 0 & 0
\end{array}\right]\left\{\begin{array}{l}
\varphi_{x} \\
\varphi_{y} \\
\varphi_{z}
\end{array}\right\}
\end{aligned}
$$

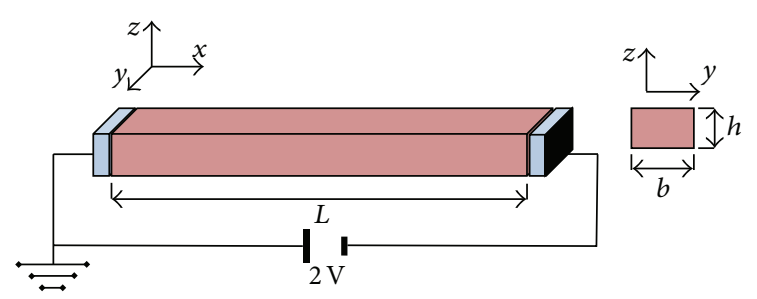

FIGURE 1: A piezoelectric microbeam with rectangular cross-section and its coordinate system.

$$
\begin{aligned}
\left\{\begin{array}{l}
D_{x} \\
D_{y} \\
D_{z}
\end{array}\right\}= & {\left[\begin{array}{cccccc}
0 & 0 & 0 & 0 & e_{5} & 0 \\
0 & 0 & 0 & e_{4} & 0 & 0 \\
e_{1} & e_{2} & e_{3} & 0 & 0 & 0
\end{array}\right]\left\{\begin{array}{l}
\varepsilon_{x x} \\
\varepsilon_{y y} \\
\varepsilon_{z z} \\
\varepsilon_{y z} \\
\varepsilon_{z x} \\
\varepsilon_{x y}
\end{array}\right\} } \\
& +\left[\begin{array}{ccc}
\eta_{1} & 0 & 0 \\
0 & \eta_{2} & 0 \\
0 & 0 & \eta_{3}
\end{array}\right]\left\{\begin{array}{l}
\varphi_{x} \\
\varphi_{y} \\
\varphi_{z}
\end{array}\right\},
\end{aligned}
$$

in which $E_{i j}, e_{i}$, and $\eta_{i}$ represent the matrices of elastic, piezoelectric, and dielectric constants, respectively. In the piezoelectric materials, the electric-field components are determined by the electric potential $\Phi$ as

$$
\varphi_{x}=-\frac{\partial \Phi}{\partial x}, \quad \varphi_{z}=-\frac{\partial \Phi}{\partial z}, \quad \varphi_{y}=0
$$

The poling direction of the piezoelectric medium is along the positive $x$-axis, where $(x, z)$ is a rectangular Cartesian coordinate system as shown in Figure 1.

Numerical simulations in the literature reveal that the electric potential along the microbeam ( $x$-axis) except in the vicinity of two ends is almost constant which results in $\varphi_{x} \leq$ $\varphi_{z}[40]$.

From (16), the electric displacements and stresses for a piezoelectric microbeam are obtained as follows:

$$
\begin{aligned}
D_{x} & =e_{5} \varepsilon_{x z}+\eta_{11} \varphi_{x}, \\
D_{z} & =e_{1} \varepsilon_{x x}+\eta_{33} \varphi_{z}, \\
\sigma_{x} & =E_{11} \varepsilon_{x x}-e_{1} \varphi_{z}, \\
\sigma_{x z} & =\tau_{x z}=E_{55} \varepsilon_{x z}-e_{5} \varphi_{x} .
\end{aligned}
$$

It is mentioned that $\varphi_{x} \leq \varphi_{z} ; \eta_{11}$ and $\eta_{33}$ are on the same order, so the electric displacement $D_{x}$ in comparison with $D_{z}$ is insignificant.

In the absence of electric charges, electrostatic equilibrium condition is represented as follows [31]:

$$
\frac{\partial D_{x}}{\partial x}+\frac{\partial D_{z}}{\partial z}=0 .
$$

For Euler-Bernoulli beam model, by substituting (6), (8), (18), and (19) into (20) and with neglecting the electric 


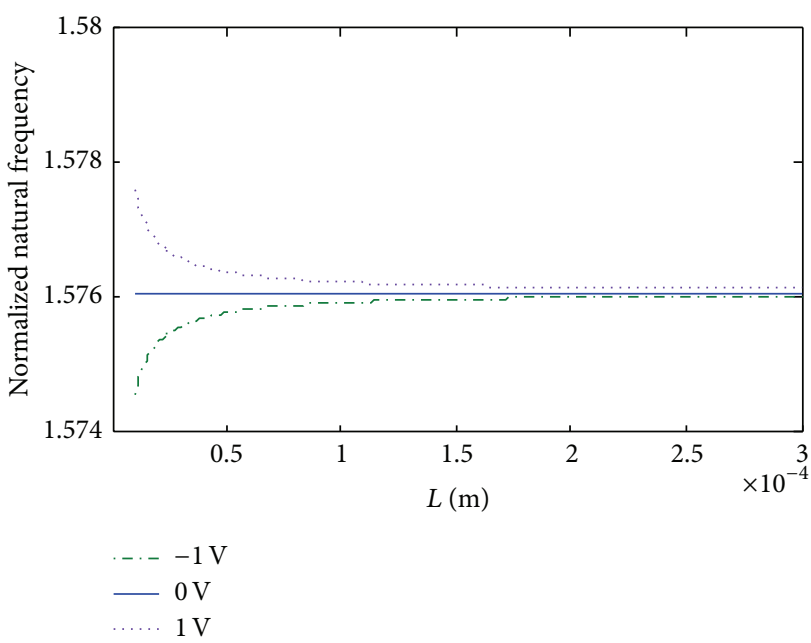

(a)

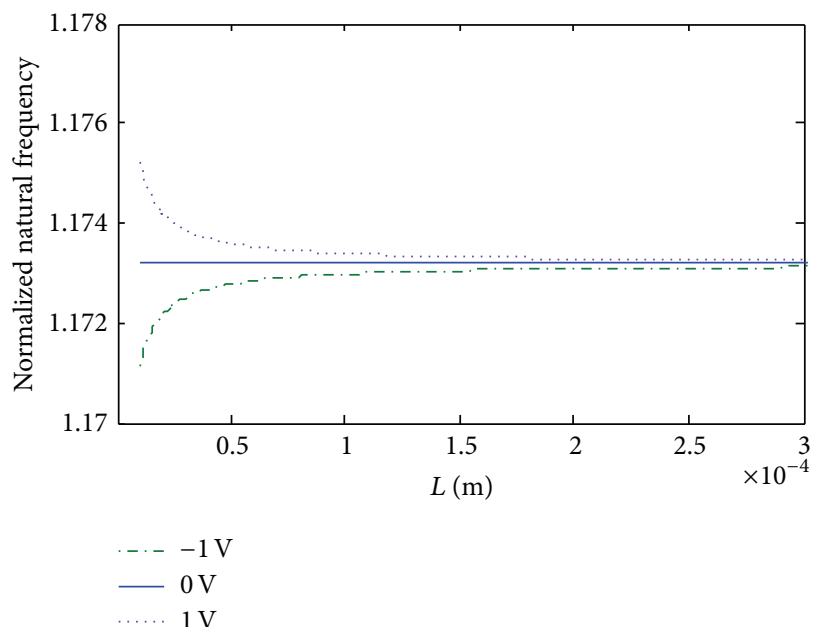

(c)

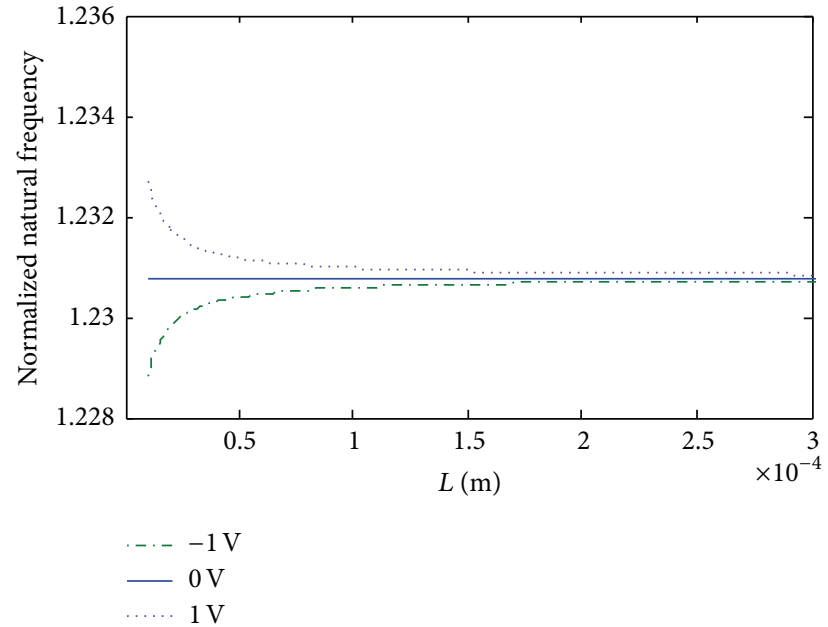

(b)

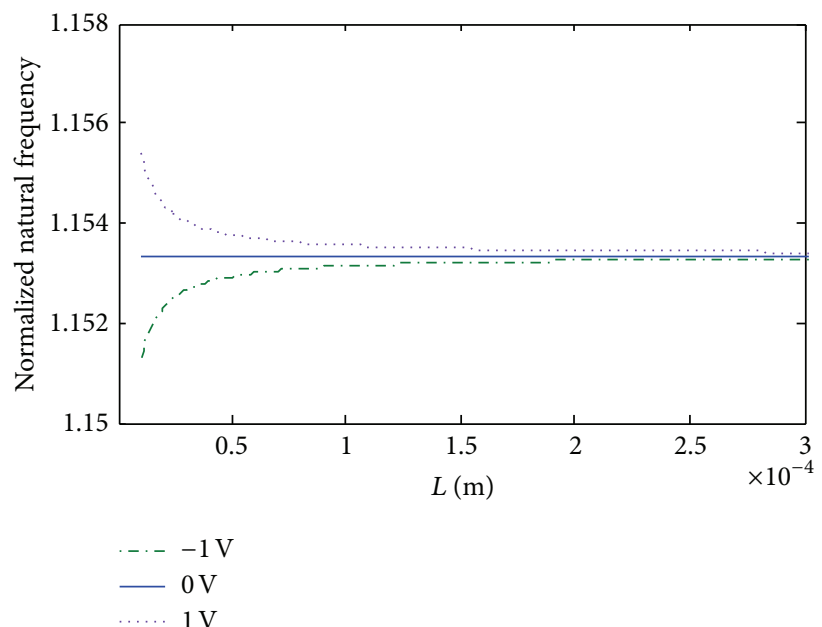

(d)

FIGURE 2: Variation of the normalized natural frequency of Euler-Bernoulli microbeam with length for different values of voltage $(L / h=20)$ : (a) $h / l=2$, (b) $h / l=5$, (c) $h / l=10$, and (d) $l=0$.

displacement $D_{x}$ in comparison with $D_{z}$, an ordinary differential equation (ODE) is obtained. The expression for electric potential as the solution of the ODE is obtained as follows $[41,42]$ :

$$
\Phi=-\left(\frac{e_{1}}{\lambda_{33}}\right)\left(\frac{z^{2}}{2}\right)\left(\frac{\partial^{2} w}{\partial x^{2}}\right)+C_{1} z+C_{2}
$$

Assuming $\Phi(-h / 2)=0$ and $\Phi(h / 2)=2 V$ gives

$$
\Phi=-\left(\frac{e_{1}}{2 \lambda_{33}}\right)\left(z^{2}-\frac{h^{2}}{4}\right)\left(\frac{\partial^{2} w}{\partial x^{2}}\right)+\left(1+\frac{2 z}{h}\right) V .
$$

For the Timoshenko beam model, substitution of (7), (9), (18), and (19) into (20) yields an ODE, and, by solving it, the electric potential is represented as

$$
\begin{aligned}
\Phi= & {\left[\left(\frac{e_{5}+2 e_{1}}{4 \lambda_{33}}\right)\left(\frac{\partial \psi}{\partial x}\right)+\left(\frac{e_{5}}{4 \lambda_{33}}\right)\left(\frac{\partial^{2} w}{\partial x^{2}}\right)\right] z^{2} } \\
& +C_{1} z+C_{2} .
\end{aligned}
$$

Without loss of generality, assuming $\Phi(-h / 2)=0$ and $\Phi(h / 2)=2 V$ leads to

$$
\begin{aligned}
\Phi= & \left(\frac{e_{5}+2 e_{1}}{4 \lambda_{33}}\right)\left(z^{2}-\frac{h^{2}}{4}\right)\left(\frac{\partial \psi}{\partial x}\right)+\left(\frac{e_{5}}{4 \lambda_{33}}\right) \\
& \times\left(z^{2}-\frac{h^{2}}{4}\right)\left(\frac{\partial^{2} w}{\partial x^{2}}\right)+\left(1+\frac{2 z}{h}\right) V .
\end{aligned}
$$

For the Euler-Bernoulli beam model, from (8) and (18)(22), the nonzero components of stress tensor can be written as follows:

$$
\sigma_{x}=-z(\lambda+2 \mu)\left(\frac{\partial^{2} w}{\partial x^{2}}\right)-z\left(\frac{e_{1}^{2}}{\lambda_{33}}\right)\left(\frac{\partial^{2} w}{\partial x^{2}}\right)+2 e_{1} \frac{V}{h}
$$


The resultant axial force, which is induced by the applied electric potential, is in the form

$$
N_{x x}=b \int_{-h / 2}^{h / 2} \sigma_{x} d z=2 V b e_{1}
$$

For the Timoshenko beam model, from (9), (18)-(20), (23), and (24), the nonzero components of stress tensor are obtained as

$$
\begin{aligned}
\sigma_{x}= & z(\lambda+2 \mu)\left(\frac{\partial \psi}{\partial x}\right)+z\left(\frac{e_{1} e_{5}}{2 \lambda_{33}}+\frac{e_{1}^{2}}{\lambda_{33}}\right)\left(\frac{\partial \psi}{\partial x}\right) \\
& +z\left(\frac{e_{1} e_{5}}{2 \lambda_{33}}\right)\left(\frac{\partial^{2} w}{\partial x^{2}}\right)+2 e_{1} \frac{V}{h}, \\
\sigma_{x z}= & \sigma_{z x}=\tau_{x z}=2 k_{s} \mu\left(\frac{1}{2} \psi(x, t)+\frac{1}{2} \frac{\partial w}{\partial x}\right),
\end{aligned}
$$

where $K_{s}$ is the shear coefficient which for rectangular crosssection is $K_{s}=5(1+v) /(6+5 v)$.

The resultant axial force is given by

$$
N_{x x}=b \int_{-h / 2}^{h / 2} \sigma_{x x} d z=2 V b e_{1} .
$$

By using (8), (1)-(5), (11), and (14), the expressions for the elastic and kinetic energies of the Euler-Bernoulli beam are obtained in the forms

$$
\begin{gathered}
U=\frac{1}{2} \int_{0}^{L} \int\left(\sigma_{i j} \varepsilon_{i j}+m_{i j} x_{i j}\right) d A d x \\
=\int_{0}^{L} \int_{A}\left[\left(-(\lambda+2 \mu) z\left(\frac{\partial^{2} w}{\partial x^{2}}\right)\right.\right. \\
\left.-z\left(\frac{e_{1}^{2}}{\lambda_{33}}\right)\left(\frac{\partial^{2} w}{\partial x^{2}}\right)+2 e_{1} \frac{V}{h}\right) \\
\left.\quad \times\left(-\frac{1}{2} z \frac{\partial^{2} w}{\partial x^{2}}\right)+\left(\frac{1}{2} \mu l^{2}\right)\left(\frac{\partial^{2} w}{\partial x^{2}}\right)\right] d A d x, \\
T=\frac{1}{2} \int_{0}^{L} \int_{A} \rho\left[\left(\frac{\partial u_{x}}{\partial t}\right)^{2}+\left(\frac{\partial u_{z}}{\partial t}\right)^{2}\right] d A d x \\
=\frac{1}{2} \int_{0}^{L} \int \rho\left[z^{2}\left(\frac{\partial}{\partial t} \frac{\partial w}{\partial x}\right)^{2}+\left(\frac{\partial w}{\partial t}\right)^{2}\right] d A d x .
\end{gathered}
$$

Also, external work is calculated as follows:

$$
W^{\mathrm{ext}}=\frac{1}{2} \int_{0}^{L} N_{x x}\left(\frac{\partial w}{\partial x}\right)^{2} d x
$$

The Hamilton principle, which is utilized to derive the governing equations of motion, is in the form

$$
\int_{t_{1}}^{t_{2}}\left(\delta T-\delta U+\delta W^{\mathrm{ext}}\right) d t=0 .
$$

Using (31), the governing equations and corresponding boundary conditions are obtained as

$$
\begin{gathered}
\left((\lambda+2 \mu) I+\frac{e_{1}^{2} I}{\lambda_{33}}+A \mu l^{2}\right)\left(\frac{\partial^{4} w}{\partial x^{4}}\right) \\
-2 \operatorname{Vbe}_{1}\left(\frac{\partial^{2} w}{\partial x^{2}}\right)=\rho A \frac{\partial^{2} w}{\partial t^{2}},
\end{gathered}
$$

$$
\begin{gathered}
\left.\frac{\partial}{\partial x}\left[M_{x x}+Y_{x y}\right]\right|_{x=0} ^{x=L}=0 \quad \text { or }\left.\quad \delta W\right|_{x=0} ^{x=L}=0, \\
{\left.\left[M_{x x}+Y_{x y}\right]\right|_{x=0} ^{x=L}=0 \quad \text { or }\left.\quad \delta\left(\frac{\partial w}{\partial x}\right)\right|_{x=0} ^{x=L}=0,}
\end{gathered}
$$

where

$$
\begin{aligned}
M_{x x} & =\int z \sigma_{x x} d A=-I\left((\lambda+2 \mu)+\frac{e_{1}^{2}}{\lambda_{33}}\right)\left(\frac{\partial^{2} w}{\partial x^{2}}\right), \\
Y_{x y} & =\int m_{x y} d A=-A \mu l^{2}\left(\frac{\partial^{2} w}{\partial x^{2}}\right) .
\end{aligned}
$$

For the Timoshenko beam model, by using (1)-(5), (9), (12), and (15), the expressions for the elastic and kinetic energies are obtained as follows:

$$
\begin{gathered}
U=\frac{1}{2} \int_{0}^{L} \int\left(\sigma_{i j} \varepsilon_{i j}+m_{i j} \chi_{i j}\right) d A d x \\
=\int_{0}^{L} \int_{A}\left[2 \mu k_{s}\left(\frac{1}{2} \psi(x, t)+\frac{1}{2} \frac{\partial w}{\partial x}\right)^{2}\right. \\
+\frac{1}{2}\left((\lambda+2 \mu) z\left(\frac{\partial \psi}{\partial x}\right)+z\left(\frac{e_{1} e_{5}}{2 \lambda_{33}}+\frac{e_{1}^{2}}{\lambda_{33}}\right)\right. \\
\times\left(z \frac{\partial \psi}{\partial x}\right)^{2}+2 \mu l^{2} \\
\left.\times\left(\frac{\partial \psi}{\partial x}\right)+z\left(\frac{e_{1} e_{5}}{2 \lambda_{33}}\right)\left(\frac{\partial^{2} w}{\partial x^{2}}\right)+2 e_{1} \frac{V}{h}\right) \\
T=\frac{1}{2} \int_{0}^{L} \int_{A} \rho\left[\left(\frac{\partial u_{x}}{\partial t}\right)^{2}+\left(\frac{\partial u_{z}}{\partial t}\right)^{2}\right] d A d x \\
=\frac{1}{2} \int_{0}^{L} \int \rho\left[z^{2}\left(\frac{\partial \psi}{\partial t}\right)^{2}+\left(\frac{\partial w}{\partial t}\right)^{2}\right] d A d x . \\
\left.\int^{2}\right] d A d x,
\end{gathered}
$$

In this model, the external work is computed as

$$
W^{\mathrm{ext}}=\frac{1}{2} \int_{0}^{L} N_{x x}\left(\frac{\partial w}{\partial x}\right)^{2} d x
$$


Using (31), the governing equations and corresponding boundary conditions are obtained in the forms

$$
\begin{aligned}
& k_{s} \mu A\left(\frac{\partial^{2} w}{\partial x^{2}}+\frac{\partial \psi}{\partial x}\right)-2 V b e_{1}\left(\frac{\partial^{2} w}{\partial x^{2}}\right)+\frac{1}{4} \mu A l^{2} \\
& \times\left(\frac{\partial^{3} \psi}{\partial x^{3}}-\frac{\partial^{4} w}{\partial x^{4}}\right)-\left(\frac{e_{1} e_{5} I}{4 \lambda_{33}}\right)\left(\frac{\partial^{3} \psi}{\partial x^{3}}\right)=\rho A \frac{\partial^{2} w}{\partial t^{2}} \\
& \left((\lambda+2 \mu) I+\left(\frac{2 e_{1}^{2}+e_{1} e_{5}}{2 \lambda_{33}}\right) I+\frac{1}{4} \mu A l^{2}\right)\left(\frac{\partial^{2} \psi}{\partial x^{2}}\right) \\
& +\left(\frac{e_{1} e_{5} I}{4 \lambda_{33}}\right)\left(\frac{\partial^{3} w}{\partial x^{3}}\right)-k_{s} \mu A\left(\frac{\partial w}{\partial x}+\psi(x, t)\right) \\
& \quad-\frac{1}{4} \mu A l^{2}\left(\frac{\partial^{3} w}{\partial x^{3}}\right)=\rho I\left(\frac{\partial^{2} \psi}{\partial t^{2}}\right), \\
& {\left.\left[M_{x x}+Q_{x}+\frac{1}{2} Y_{x y}\right]\right|_{x=0} ^{x=L}=0} \\
& \text { or }\left.\delta \psi\right|_{x=0} ^{x=L}=0=0, \\
& {\left.\left[Q_{x}+\frac{1}{2} Y_{x y}-I\left((\lambda+2 \mu)+\frac{2 e_{1}^{2}+e_{1} e_{5}}{2 \lambda_{33}}\right) \frac{\partial^{2} \psi}{\partial x^{2}}\right]\right|_{x=0} ^{x=L}=0} \\
& \text { or }\left.\delta w\right|_{x=0} ^{x=L}=0, \\
& \left.\quad \delta\left(\frac{\partial w}{\partial x}\right)\right|_{x=0} ^{x=L}=0, \\
& {\left.\left[\frac{1}{2} Y_{x y}+\left(\frac{e_{1} e_{5} I}{2 \lambda_{33}}\right)\left(\frac{\partial \psi}{\partial x}\right)\right]\right|_{x=0} ^{x=L}=0}
\end{aligned}
$$

where

$$
\begin{aligned}
& M_{x x}=\int z \sigma_{x x} d A= I\left((\lambda+2 \mu)+\frac{2 e_{1}^{2}+e_{1} e_{5}}{2 \lambda_{33}}\right) \\
& \times\left(\frac{\partial \psi}{\partial x}\right)+\left(\frac{e_{1} e_{5} I}{2 \lambda_{33}}\right)\left(\frac{\partial^{2} w}{\partial x^{2}}\right), \\
& Y_{x y}=\int m_{x y} d A= \frac{1}{2} A \mu l^{2}\left(\frac{\partial \psi}{\partial x}-\frac{\partial^{2} w}{\partial x^{2}}\right), \\
& Q_{x}=\int \sigma_{x z} d A=k_{s} \mu A\left(\frac{\partial w}{\partial x}+\psi(x, t)\right) .
\end{aligned}
$$

\section{Navier Solution of the Governing Equations}

In order to solve the governing equations of motion, the Navier solution is utilized for both Euler-Bernoulli and Timoshenko beam models and the exact expressions of natural frequencies are derived. For the Euler-Bernoulli beam model, assume the periodic solution is as follows:

$$
w(x, t)=W(x) e^{i \omega t},
$$

where $\omega$ is the natural frequency of vibration. Substitution of (44) into (32) gives

$$
r_{1}\left(\frac{d^{4} W(x)}{d x^{4}}\right)+r_{2}\left(\frac{d^{4} W(x)}{d x^{4}}\right)-r_{3}(W(x))=0,
$$

where

$$
\begin{gathered}
r_{1}=\left((\lambda+2 \mu) I+\frac{e_{1}^{2} I}{\lambda_{33}}+A \mu l^{2}\right), \quad r_{2}=-2 b V e_{1}, \\
r_{3}=\rho A \omega^{2} .
\end{gathered}
$$

The general solution is in the form

$$
W(x)=a_{1} \sin \alpha x+a_{2} \cos \alpha x+a_{3} \sin \beta x+a_{4} \cos \beta x,
$$

where

$$
\begin{aligned}
& \alpha^{2}=\frac{1}{2 r_{1}}\left(r_{2}+\sqrt{r_{2}^{2}+4 r_{1} r_{3}}\right), \\
& \beta^{2}=\frac{1}{2 r_{1}}\left(-r_{2}+\sqrt{r_{2}^{2}+4 r_{1} r_{3}}\right),
\end{aligned}
$$

and $a_{1}$ to $a_{4}$ are the integration constants which can be defined by imposing the problem boundary conditions. For a simply supported piezoelectric microbeam, the expression for $n$th natural frequencies of vibration is obtained as follows:

$$
\begin{aligned}
& \omega=(n \pi) \\
& \times\left(\left(-\rho A\left(-\pi^{2}\left((\lambda+2 \mu) I+\frac{e_{1}^{2} I}{\lambda_{33}}+A \mu l^{2}\right) n^{2}\right.\right.\right. \\
& \left.\left.\quad+2 b e_{1} V L^{2}\right)\right)^{1 / 2} \\
& \left.\quad \times\left(\rho A L^{2}\right)^{-1}\right) .
\end{aligned}
$$

For the Timoshenko beam model, assume the periodic solutions are as follows:

$$
\begin{aligned}
& w(x, t)=c_{1} \sin \left(\frac{n \pi x}{L}\right) e^{i \omega t}, \\
& \psi(x, t)=c_{2} \cos \left(\frac{n \pi x}{L}\right) e^{i \omega t} .
\end{aligned}
$$

Substituting (50) and (51) into (38) and (39) and then rearranging them in matrix form yield

$$
\left[\begin{array}{ll}
A_{11} & A_{12} \\
A_{21} & A_{22}
\end{array}\right]\left[\begin{array}{l}
c_{1} \\
c_{2}
\end{array}\right]=\left[\begin{array}{l}
0 \\
0
\end{array}\right]
$$




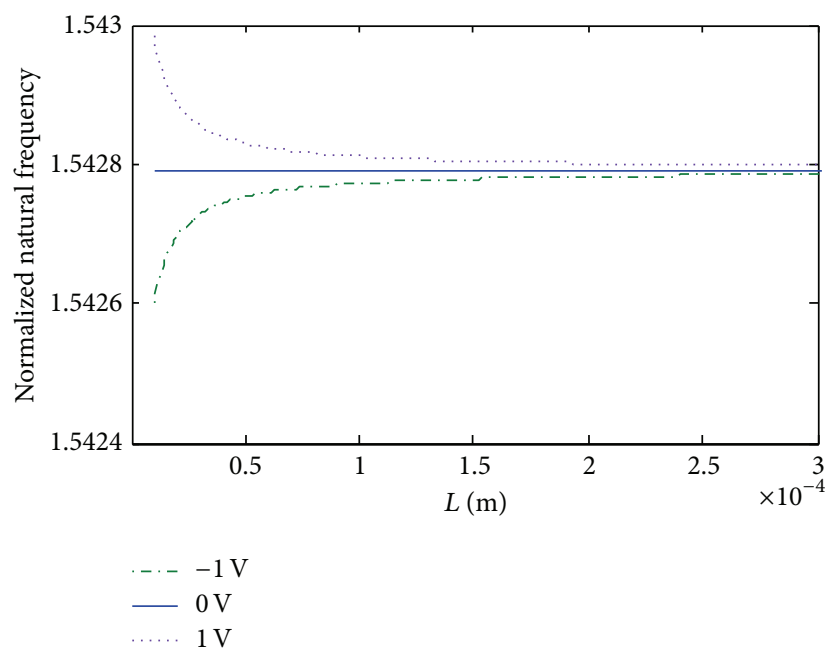

(a)

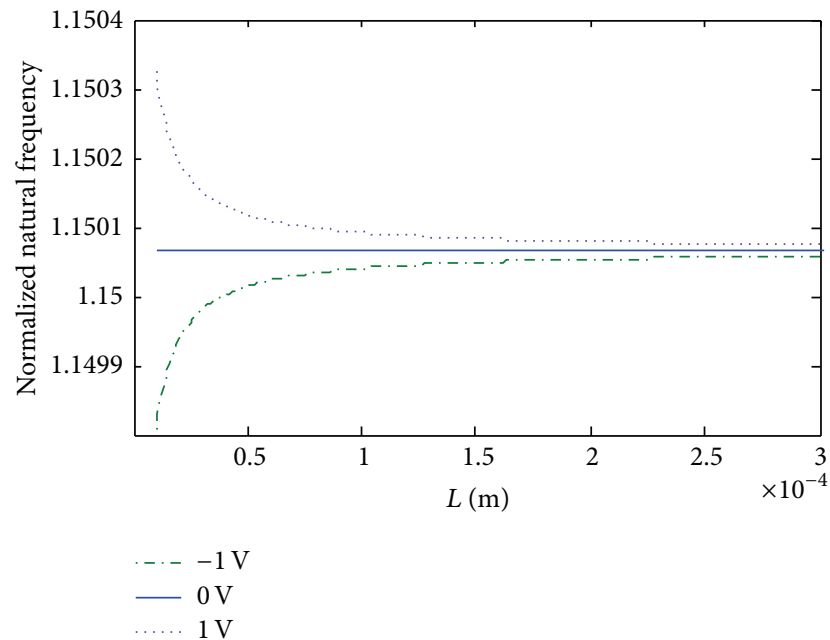

(c)

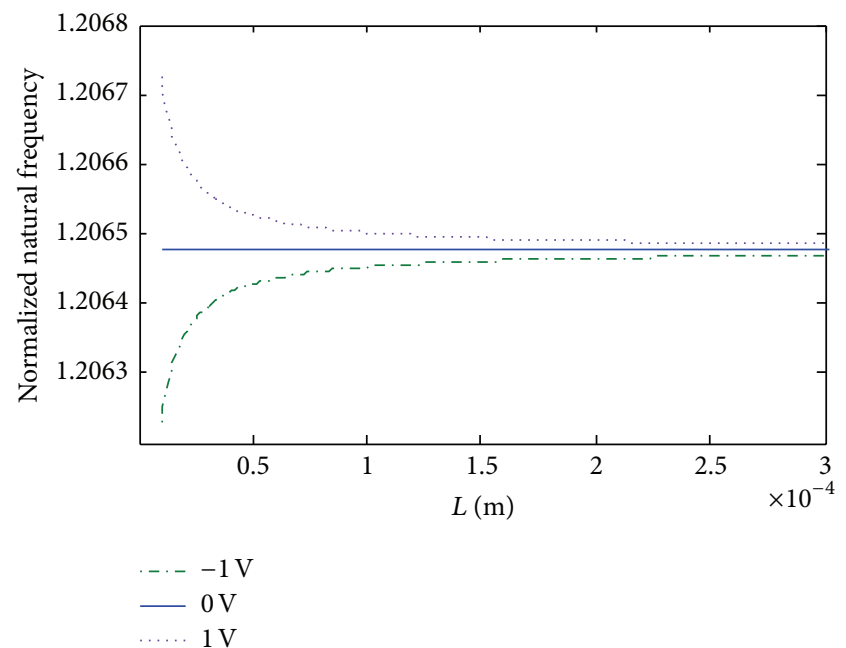

(b)

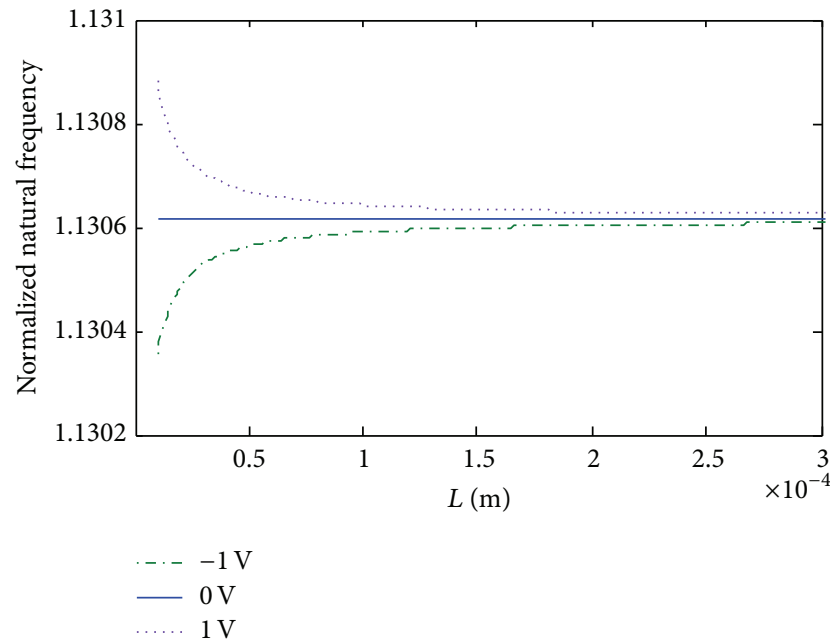

(d)

FiguRE 3: Variation of the normalized natural frequency of Timoshenko microbeam with length for different values of voltage $(L / h=10)$ : (a) $h / l=2$, (b) $h / l=5$, (c) $h / l=10$, and (d) $l=0$.

where

$$
\begin{gathered}
A_{11}=\frac{1}{4} \frac{\mu A l^{2} n^{2} \pi^{2}}{L^{3}}-\frac{1}{4} \frac{e_{1} e_{5} I^{3} \pi^{3}}{\lambda_{33} L^{3}}-\frac{k_{s} \mu A n \pi}{L}, \\
A_{12}=-k_{s} \mu A \\
-\left(\left((\lambda+2 \mu) I+\left(\frac{2 e_{1}^{2}+e_{1} e_{5}}{2 \lambda_{33}}\right) I+\frac{1}{4} \mu A l^{2}\right) n^{2} \pi^{2}\right. \\
\left.\quad \times\left(L^{2}\right)^{-1}\right)+\rho I \omega^{2}, \\
A_{21}=-\frac{k_{s} \mu A n^{2} \pi^{2}}{L^{2}}+\rho A \omega^{2}+\frac{2 V b e_{1} n^{2} \pi^{2}}{L^{2}}-\frac{1}{4} \frac{\mu A l^{2} n^{4} \pi^{4}}{L^{4}}, \\
A_{22}=\frac{1}{4} \frac{\mu A l^{2} n^{3} \pi^{3}}{L^{3}}-\frac{1}{4} \frac{1}{4} \frac{e_{1} e_{5} I n^{3} \pi^{3}}{\lambda_{33} L^{3}}-\frac{k_{s} \mu A n \pi}{L} .
\end{gathered}
$$

For a nontrivial solution of (52), the determinant of the coefficient matrix must be equal to zero that yields a characteristic equation with the eigenvalues which are the $n$th natural frequencies of vibration for a simply supported Timoshenko beam. The expression for the natural frequency of this beam model is given in the appendix.

\section{Results and Discussion}

In this section, numerical results are presented in order to demonstrate the influences of piezoelectricity and size effects on the vibration behavior of simply supported piezoelectric microbeams. The material properties of the piezoelectric microbeam are given in Table 1 [39]. Also, microbeam's height $h$ and its width $b$ are assumed to be equal $(b=h)$.

The natural frequency is normalized with respect to the frequency of classical Euler-Bernoulli beam model in the form $\omega_{0}=(n \pi / L)^{2} \sqrt{E I / \rho A}$. Also, the results are given for the first mode of vibration $(n=1)$. 

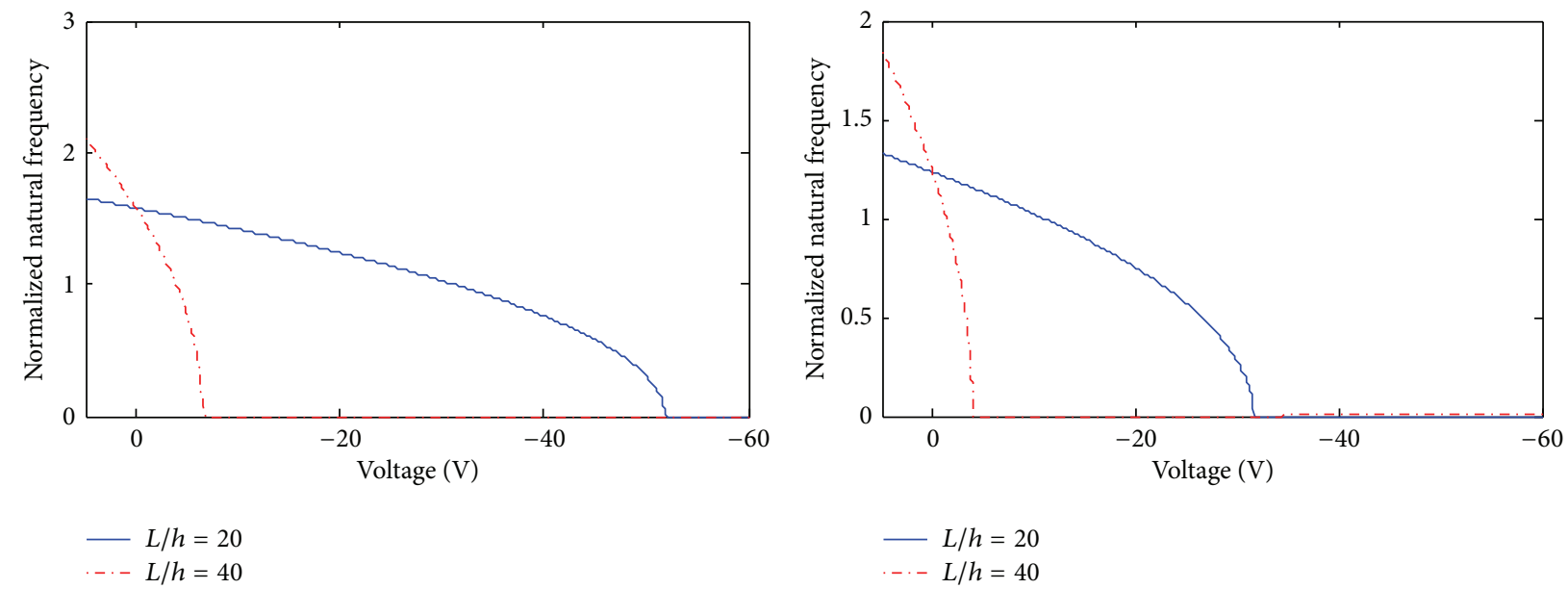

(a)
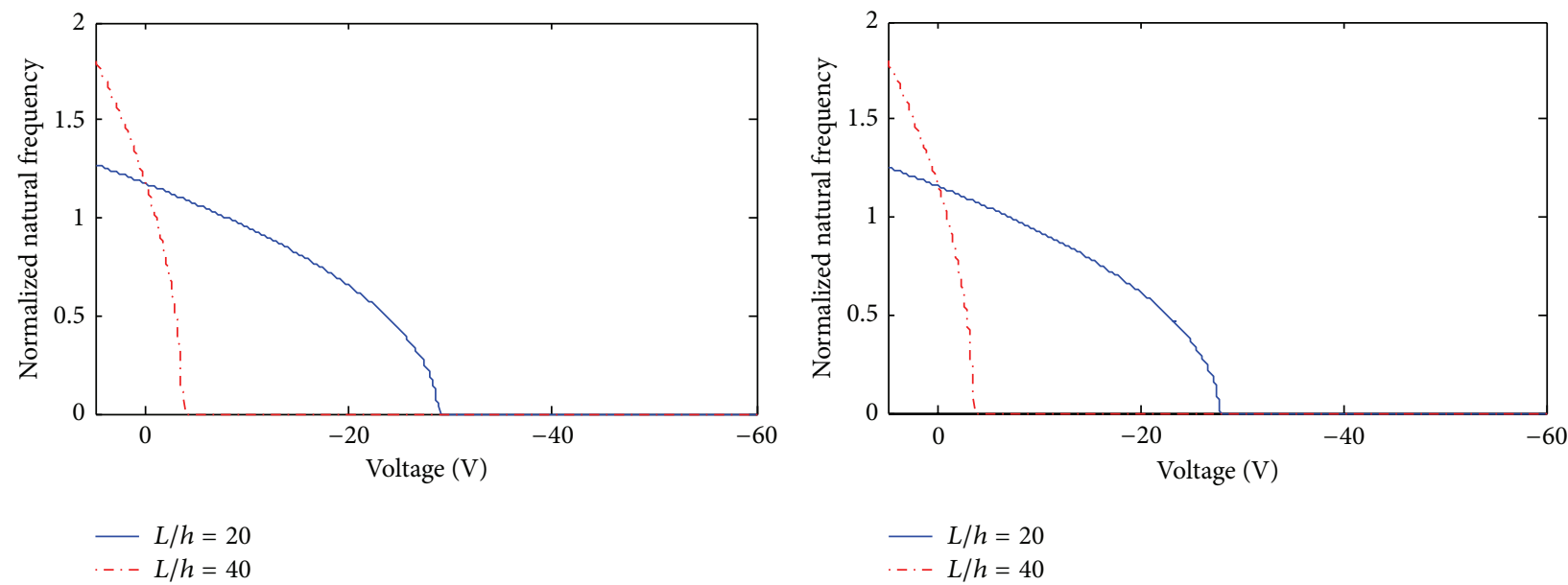

(c)

(d)

FIGURE 4: Variation of the normalized natural frequency of Euler-Bernoulli microbeam with voltage for different values of $L / h$ : (a) $h / l=2$, (b) $h / l=5$, (c) $h / l=10$, and (d) $l=0$.

TABLE 1: Material properties of piezoelectric microbeam constituents [39].

\begin{tabular}{lcccccc}
\hline $\begin{array}{l}E_{11}=\lambda+2 \mu \\
(\mathrm{GPa})\end{array}$ & $\nu$ & $\begin{array}{c}E_{55}=\mu \\
(\mathrm{GPa})\end{array}$ & $\begin{array}{c}E_{13}=\lambda \\
(\mathrm{GPa})\end{array}$ & $\begin{array}{c}e_{1} \\
\left(\mathrm{C} / \mathrm{m}^{2}\right)\end{array}$ & $\begin{array}{c}e_{5} \\
\left(\mathrm{C} / \mathrm{m}^{2}\right)\end{array}$ & $\begin{array}{c}\lambda_{33} \\
(\mathrm{~F} / \mathrm{m})\end{array}$ \\
\hline \multicolumn{1}{c}{207} & 0.349 & 44.6 & 106.1 & -0.51 & -0.45 & $-7.88 \times 10^{-11}$ \\
\hline
\end{tabular}

Figure 2 shows the variation of the normalized natural frequency of the Euler-Bernoulli microbeam with the length of beam for different values of voltage ( $V=0, \pm 1$ volt) and different values of $h / l$ and with the assumption of $L / h=20$. Similar graphs for the Timoshenko microbeam are displayed in Figure 3 by assuming that $L / h=10$. It is clear that, by increasing the length, the natural frequency for the negative values of voltage increases and for the positive values decreases. As it can be seen from these figures, for a given value of length, by increasing the value of thicknessto-length scale parameter ratio $h / l$, the natural frequency decreases. In addition, it can be found from these figures that, for all values of $h / l$, the value of normalized natural frequency is higher than the one obtained from the classical theory $(l=0)$. For short microbeams, the effects of piezoelectricity and size effects on the normalized natural frequency are more prominent. The results of the normalized natural frequency for the negative and positive values of voltage are converged after a specific length of beam and the influences of piezoelectricity vanish.

Figure 4 demonstrates the variation of the normalized natural frequency of Euler-Bernoulli microbeam with voltage for different values of length-to-thickness ratio $(L / h=20$ and 40) and different values of $h / l$. Similar graphs for the Timoshenko microbeam are shown in Figure 5 for different values of length-to-thickness ratio $(L / h=10,20$, and 40). As it can be observed from these figures, increasing the value of length-to-thickness ratio leads to the higher values of the normalized natural frequency and, also in the higher values of voltage, the normalized natural frequency 


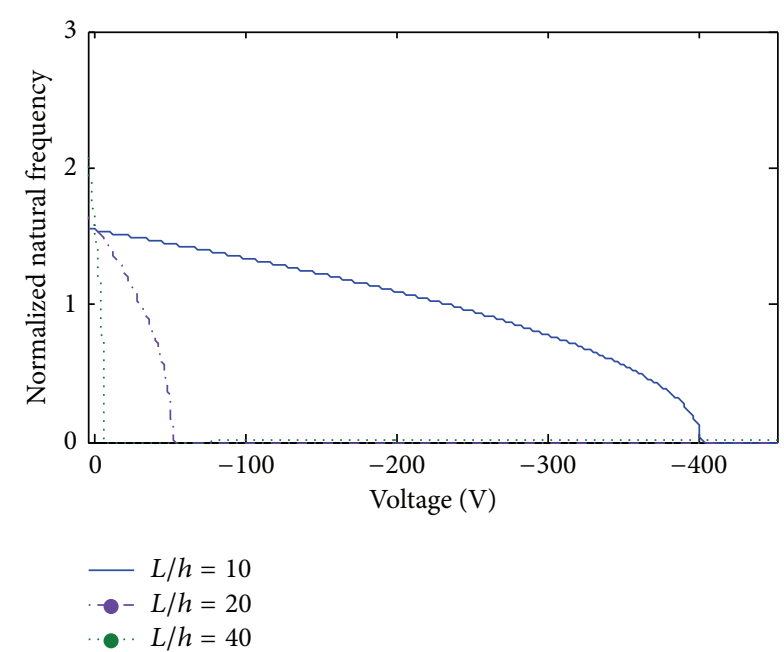

(a)

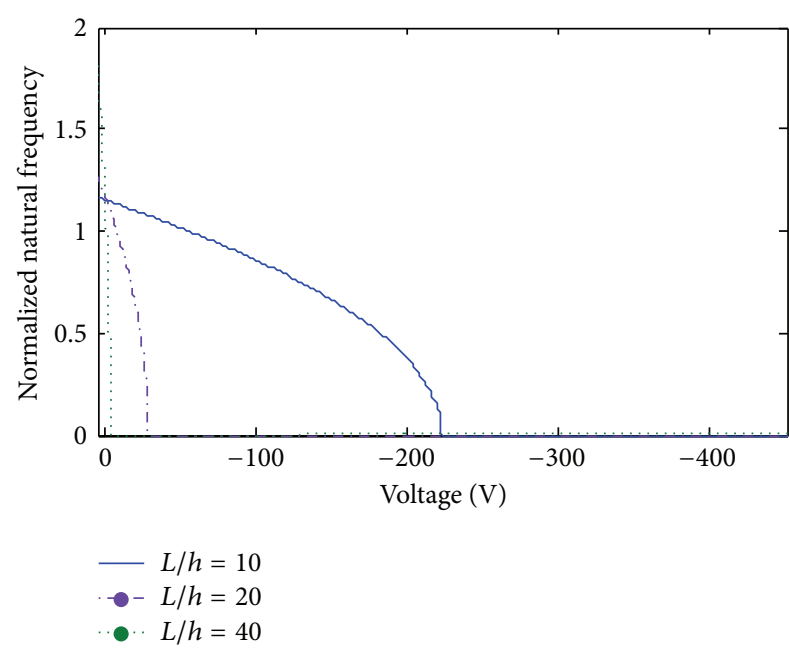

(c)

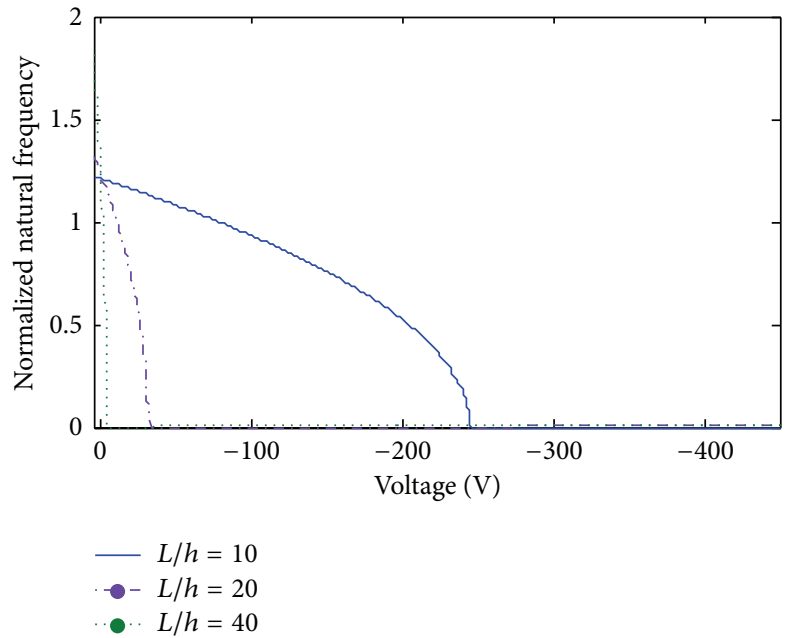

(b)

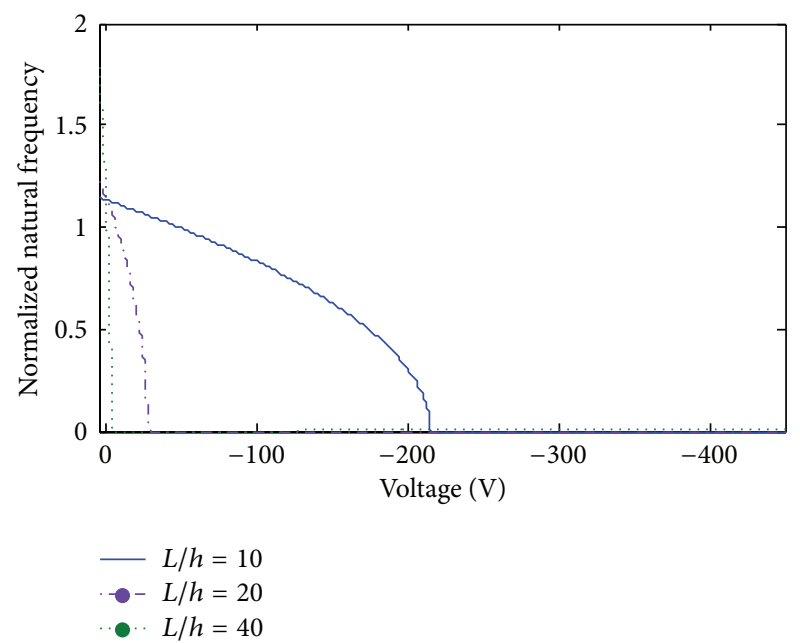

(d)

FIGURE 5: Variation of the normalized natural frequency of Timoshenko microbeam with voltage for different values of $L / h$ : (a) $h / l=2$, (b) $h / l=5$, (c) $h / l=10$, and (d) $l=0$.

becomes zero and buckling occurs. For the Timoshenko beam model, the influences of length-to-thickness ratio, voltage, and length scale parameter are similar to those in the Euler-Bernoulli beam model. In both beam models, the influences of piezoelectricity increase by the increment in length-to-thickness ratio.

Figure 6 presents the variation of the normalized natural frequency of Euler-Bernoulli and Timoshenko microbeams with the length for different values of $l / h$ and with the assumption of $L / h=40$. In this figure, the frequency of EulerBernoulli beam model has been compared with the frequency of the Timoshenko beam model. As it can be seen from this figure, the Euler-Bernoulli beam model tends to overestimate the natural frequencies as compared to the Timoshenko beam model. In addition, the comparison between two graphs demonstrates that increasing the value of thickness-to-length scale parameter ratio causes the better agreement between two curves.

\section{Conclusion}

In this paper, an exact solution was obtained for the vibration analysis of piezoelectric microbeams on the basis of the modified couple stress theory using both Euler-Bernoulli and Timoshenko beam models. First, the electric-field equations and the governing equations of motion were derived using Hamilton's principle. Then, the Navier method was utilized for the simply supported piezoelectric microbeams to solve the governing equations and to achieve an exact expression for natural frequencies. By using the normalized natural frequencies, the effects of piezoelectricity and small scale for both beam models were illustrated. The numerical results showed that, for the slender microbeams with larger length-to-thickness ratio, the effect of piezoelectricity is more considerable and, by increasing the length of microbeam, piezoelectricity and size effects gradually vanish. By increasing the value of thickness-to-length scale parameter ratio, the normalized 


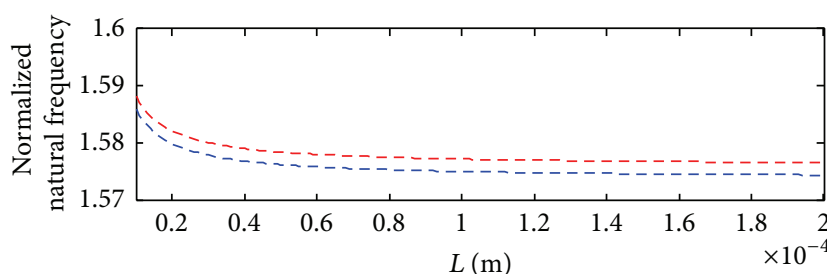

- - - Euler-Bernoulli beam model - - - Timoshenko beam model

(a)

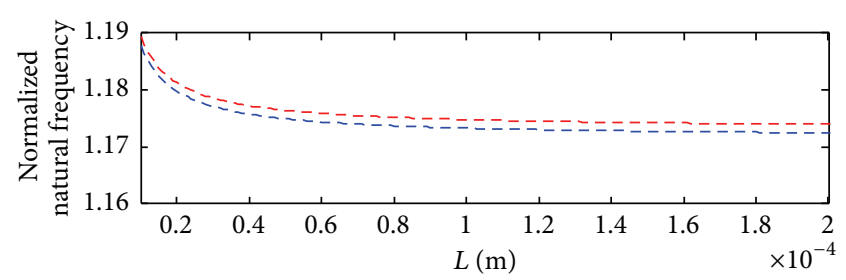

- - - Euler-Bernoulli beam model - - - Timoshenko beam model

(b)

FIGURE 6: Variation of the normalized natural frequency of Euler-Bernoulli and Timoshenko microbeams with length $(L / h=40):(a) h / l=2$ and (b) $h / l=10$.

natural frequencies decrease for both beam models. Also, it was indicated that the Euler-Bernoulli beam model tends to overestimate the natural frequencies of microbeams in comparison with the Timoshenko beam model.

\section{Appendix}

Exact expression for the natural frequency of the Timoshenko beam is as follows:

$$
\begin{aligned}
& \omega=-\frac{1}{4} \frac{1}{\lambda_{33} \rho I A L^{2}} \\
& \times\left(\sqrt { 2 } \left(\lambda_{33} \rho I A\right.\right. \\
& \times\left(\lambda_{33} k_{s} \mu A^{2} L^{4}+4 \lambda_{33} I L^{2} k_{s} \mu A n^{2} \pi^{2}\right. \\
& -8 \lambda_{33} I L^{2} b V e_{1} n^{2} \pi^{2}+4 \lambda_{33} \\
& \times\left((\lambda+2 \mu) I+\left(\frac{2 e_{1}^{2}+e_{1} e_{5}}{2 \lambda_{33}}\right) I\right. \\
& \left.+\frac{1}{4} \mu A l^{2}\right) n^{2} \pi^{2} A L^{2}+\lambda_{33} I \mu A l^{2} n^{4} \pi^{4} \\
& +\left(16 \lambda_{33}^{2} k_{s}^{2} \mu^{2} A^{4} L^{8}+16 \lambda_{33}^{2}\right. \\
& \times\left((\lambda+2 \mu) I+\left(\frac{2 e_{1}^{2}+e_{1} e_{5}}{2 \lambda_{33}}\right) I\right. \\
& \left.+\frac{1}{4} \mu A l^{2}\right)^{2} \\
& \times n^{4} \pi^{4} A^{2} L^{4}+\lambda_{33}^{2} I^{2} \mu^{2} A^{2} l^{4} n^{8} \pi^{8} \\
& +32 \lambda_{33}^{2} k_{s}^{2} \mu^{2} A^{3} L^{6} \operatorname{In}^{2} \pi^{2} \\
& +64 \lambda_{33}^{2} k_{s} \mu A^{2} L^{6} I b V e_{1} n^{2} \pi^{2} \\
& +32 \lambda_{33}^{2} k_{s} \mu A^{3} L^{6} \\
& \times\left((\lambda+2 \mu) I+\left(\frac{2 e_{1}^{2}+e_{1} e_{5}}{2 \lambda_{33}}\right) I\right.
\end{aligned}
$$

$$
\begin{gathered}
\left.+\frac{1}{4} \mu A l^{2}\right) n^{2} \pi^{2} \pi^{2} \\
-40 \lambda_{33}^{2} k_{s} \mu^{2} A^{3} L^{4} I l^{2} n^{4} \pi^{4} \\
+16 \lambda_{33}^{2} I^{2} L^{4} k_{s}^{2} \mu^{2} A^{2} n^{4} \pi^{4} \\
-64 \lambda_{33}^{2} I^{2} L^{4} k_{s} \mu A n^{4} \pi^{4} b V e_{1} \\
-32 \lambda_{33}^{2} I L^{4} k_{s} \mu A^{2} n^{4} \pi^{4} \\
\times\left((\lambda+2 \mu) I+\left(\frac{2 e_{1}^{2}+e_{1} e_{5}}{2 \lambda_{33}}\right) I\right. \\
\left.\left.\left.+32 I^{2} A^{2} L^{4} n^{4} \pi^{4} e_{1} e_{5} k_{s} \mu \lambda_{33}\right)^{1 / 2}\right)\right) \\
+8 I^{2} A^{2} L^{2} n^{6} \pi^{6} \mu l^{2} \lambda_{33} e_{1} e_{5} \\
+4 I^{3} A L^{2} n^{6} \pi^{6} e_{1}^{2} e_{5}^{2} \\
+8 \lambda_{33}^{2} I^{2} L^{2} k_{s} \mu^{2} A^{2} n^{6} \pi^{6} l^{2} \\
+64 \lambda_{33}^{2} I^{2} L^{4} b^{2} V^{2} e_{1}^{2} n^{4} \pi^{4} \\
+64 \lambda_{33}^{2} I L^{4} b V e_{1} n^{4} \pi^{4} \\
+8 \lambda_{33}^{2}\left(\left(\lambda I^{2} L^{2} b V e_{1} n^{6} \pi^{6} \mu A l^{2}\right.\right. \\
\times\left((\lambda+2 \mu) I+\left(\frac{2 e_{1}^{2}+e_{1} e_{5}}{2 \lambda_{33}}\right) I\right. \\
\left.+\frac{1}{4} \mu A l^{2}\right) A
\end{gathered}
$$




\section{Conflict of Interests}

The authors declare that there is no conflict of interests regarding the publication of this paper.

\section{References}

[1] F.-Y. Lun, P. Zhang, F.-B. Gao, and H.-G. Jia, "Design and fabrication of micro-optomechanical vibration sensor," Microfabrication Technology, vol. 120, no. 1, pp. 61-64, 2006.

[2] M. Mojahedi, M. M. Zand, and M. T. Ahmadian, "Static pull-in analysis of electrostatically actuated microbeams using homotopy perturbation method," Applied Mathematical Modelling, vol. 34, no. 4, pp. 1032-1041, 2010.

[3] M. T. Ahmadian, H. Borhan, and E. Esmailzadeh, "Dynamic analysis of geometrically nonlinear and electrostatically actuated micro-beams," Communications in Nonlinear Science and Numerical Simulation, vol. 14, no. 4, pp. 1627-1645, 2009.

[4] S. Roy, S. Furukawa, and M. Mehregany, "Determination of Young's modulus and residual stress of electroless nickel using test structures fabricated in a new surface micromachining process," Microsystem Technologies, vol. 2, no. 2, pp. 92-96, 1996.

[5] M. H. Mahdavi, A. Farshidianfar, M. Tahani, S. Mahdavi, and H. Dalir, "A more comprehensive modeling of atomic force microscope cantilever," Ultramicroscopy, vol. 109, no. 1, pp. 5460, 2008.

[6] N. A. Fleck and J. W. Hutchinson, "A phenomenological theory for strain gradient effects in plasticity," Journal of the Mechanics and Physics of Solids, vol. 41, no. 12, pp. 1825-1857, 1993.

[7] Q. Ma and D. R. Clarke, "Size dependent hardness of silver single crystals," Journal of Materials Research, vol. 10, no. 4, pp. 853-863, 1995.

[8] D. C. C. Lam, F. Yang, A. C. M. Chong, J. Wang, and P. Tong, "Experiments and theory in strain gradient elasticity," Journal of the Mechanics and Physics of Solids, vol. 51, no. 8, pp. 14771508, 2003.

[9] N. A. Fleck, G. M. Muller, M. F. Ashby, and J. W. Hutchinson, "Strain gradient plasticity: theory and experiment," Acta Metallurgica et Materialia, vol. 42, no. 2, pp. 475-487, 1994.

[10] A. C. M. Chong and D. C. C. Lam, "Strain gradient plasticity effect in indentation hardness of polymers," Journal of Materials Research, vol. 14, no. 10, pp. 4103-4110, 1999.

[11] S. Papargyri-Beskou, K. G. Tsepoura, D. Polyzos, and D. E. Beskos, "Bending and stability analysis of gradient elastic beams," International Journal of Solids and Structures, vol. 40, no. 2, pp. 385-400, 2003.

[12] A. C. Eringen, "On differential equations of nonlocal elasticity and solutions of screw dislocation and surface waves," Journal of Applied Physics, vol. 54, no. 9, pp. 4703-4710, 1983.

[13] R. D. Mindlin and H. F. Tiersten, "Effects of couple-stresses in linear elasticity," Archive for Rational Mechanics and Analysis, vol. 11, no. 1, pp. 415-448, 1962.

[14] R. A. Toupin, "Elastic materials with couple-stresses," Archive for Rational Mechanics and Analysis, vol. 11, no. 1, pp. 385-414, 1962.

[15] W. T. Koiter, "Couple-stresses in the theory of elasticityI and II," Proceedings of the Koninklijke Nederlandse Academie van Wetenschappen B: Physical Sciences, vol. 67, pp. 17-44, 1964.

[16] S. J. Zhou and Z. Q. Li, "Length scales in the static and dynamic torsion of a circular cylindrical micro-bar," Journal of Shandong University of Technology, vol. 31, no. 5, pp. 401-407, 2001.
[17] X. Kang and Z. Xi, "Size effect on the dynamic characteristic of a micro beam based on Cosserat theory," Journal of Mechanical Strength, vol. 29, no. 1, pp. 1-4, 2007.

[18] M. Asghari, M. H. Kahrobaiyan, M. Rahaeifard, and M. T. Ahmadian, "Investigation of the size effects in Timoshenko beams based on the couple stress theory," Archive of Applied Mechanics, vol. 81, no. 7, pp. 863-874, 2011.

[19] F. Yang, A. C. M. Chong, D. C. C. Lam, and P. Tong, "Couple stress based strain gradient theory for elasticity," International Journal of Solids and Structures, vol. 39, no. 10, pp. 2731-2743, 2002.

[20] S. K. Park and X.-L. Gao, "Bernoulli-Euler beam model based on a modified couple stress theory," Journal of Micromechanics and Microengineering, vol. 16, no. 11, article 015, pp. 2355-2359, 2006.

[21] H. M. Ma, X.-L. Gao, and J. N. Reddy, "A microstructuredependent Timoshenko beam model based on a modified couple stress theory," Journal of the Mechanics and Physics of Solids, vol. 56, no. 12, pp. 3379-3391, 2008.

[22] J. N. Reddy, "Microstructure-dependent couple stress theories of functionally graded beams," Journal of the Mechanics and Physics of Solids, vol. 59, no. 11, pp. 2382-2399, 2011.

[23] S. Kong, S. Zhou, Z. Nie, and K. Wang, "The size-dependent natural frequency of Bernoulli-Euler micro-beams," International Journal of Engineering Science, vol. 46, no. 5, pp. 427-437, 2008.

[24] M. Asghari, M. H. Kahrobaiyan, and M. T. Ahmadian, "A nonlinear Timoshenko beam formulation based on the modified couple stress theory," International Journal of Engineering Science, vol. 48, no. 12, pp. 1749-1761, 2010.

[25] M. Asghari, M. T. Ahmadian, M. H. Kahrobaiyan, and M. Rahaeifard, "On the size-dependent behavior of functionally graded micro-beams," Materials and Design, vol. 31, no. 5, pp. 2324-2329, 2010.

[26] M. Asghari, M. Rahaeifard, M. H. Kahrobaiyan, and M. T. Ahmadian, "The modified couple stress functionally graded Timoshenko beam formulation," Materials and Design, vol. 32, no. 3, pp. 1435-1443, 2011.

[27] W. Xia, L. Wang, and L. Yin, "Nonlinear non-classical microscale beams: static bending, postbuckling and free vibration," International Journal of Engineering Science, vol. 48, no. 12, pp. 2044-2053, 2010.

[28] Y.-G. Wang, W.-H. Lin, and N. Liu, "Nonlinear free vibration of a microscale beam based on modified couple stress theory," Physica E: Low-Dimensional Systems and Nanostructures, vol. 47, pp. 80-85, 2013.

[29] B. Gheshlaghi and S. M. Hasheminejad, "Vibration analysis of piezoelectric nanowires with surface and small scale effects," Current Applied Physics, vol. 12, no. 4, pp. 1096-1099, 2012.

[30] Z. Yan and L. Jiang, "Surface effects on the electromechanical coupling and bending behaviours of piezoelectric nanowires," Journal of Physics D: Applied Physics, vol. 44, no. 7, Article ID 075404, 2011.

[31] G.-F. Wang and X.-Q. Feng, "Effect of surface stresses on the vibration and buckling of piezoelectric nanowires," Europhysics Letters, vol. 91, no. 5, Article ID 56007, 2010.

[32] J. Zhang, C. Wang, and S. Adhikari, "Surface effect on the buckling of piezoelectric nanofilms," Journal of Physics D: Applied Physics, vol. 45, Article ID 285301, 8 pages, 2012.

[33] Z. Yan and L. Jiang, "Electromechanical response of a curved piezoelectric nanobeam with the consideration of surface effects," Journal of Physics D: Applied Physics, vol. 44, no. 36, Article ID 365301, 2011. 
[34] K. Wang and B. Wang, "Surface effects on the buckling of piezoelectric nanobeams," Advanced Materials Research, vol. 486, pp. 519-523, 2012.

[35] L.-L. Ke, Y.-S. Wang, and Z.-D. Wang, "Nonlinear vibration of the piezoelectric nanobeams based on the nonlocal theory," Composite Structures, vol. 94, no. 6, pp. 2038-2047, 2012.

[36] Y. Li, C. Chen, B. Fang, J. Zhang, and J. Song, "Postbuckling of piezoelectric nanobeams with surface effects," International Journal of Applied Mechanics, vol. 4, no. 2, Article ID 1250018, 10 pages, 2012.

[37] X. Liang and S. Shen, "Effect of electrostatic force on a piezoelectric nanobeam," Smart Materials and Structures, vol. 21, no. 1, Article ID 015001, 2012.

[38] Z. Yan and L. Y. Jiang, "The vibrational and buckling behaviors of piezoelectric nanobeams with surface effects," Nanotechnology, vol. 22, no. 24, Article ID 245703, 2011.

[39] Y. Gao and Z. L. Wang, "Electrostatic potential in a bent piezoelectric nanowire. The fundamental theory of nanogenerator and nanopiezotronics," Nano Letters, vol. 7, no. 8, pp. 24992505, 2007.

[40] Z. L. Wang and J. Song, "Piezoelectric nanogenerators based on zinc oxide nanowire arrays," Science, vol. 312, no. 5771, pp. 243246, 2006.

[41] A. Milazzo, C. Orlando, and A. Alaimo, "An analytical solution for the magneto-electro-elastic bimorph beam forced vibrations problem," Smart Materials and Structures, vol. 18, no. 8, Article ID 085012, 2009.

[42] S. V. Gopinathan, V. V. Varadan, and V. K. Varadan, "Review and critique of theories for piezoelectric laminates," Smart Materials and Structures, vol. 9, no. 1, pp. 24-48, 2000. 

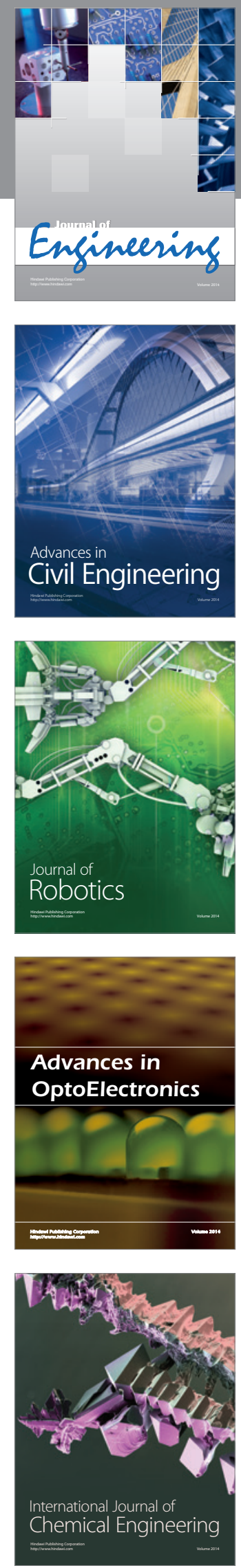

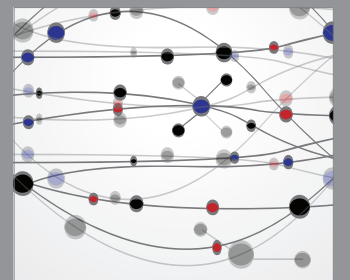

The Scientific World Journal
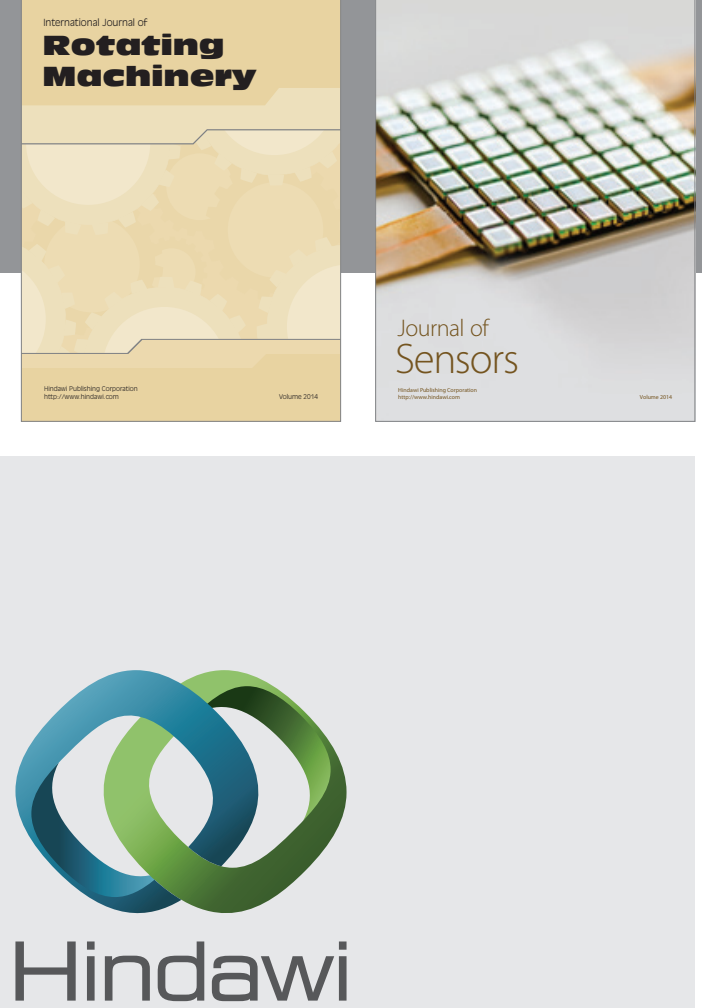

Submit your manuscripts at http://www.hindawi.com
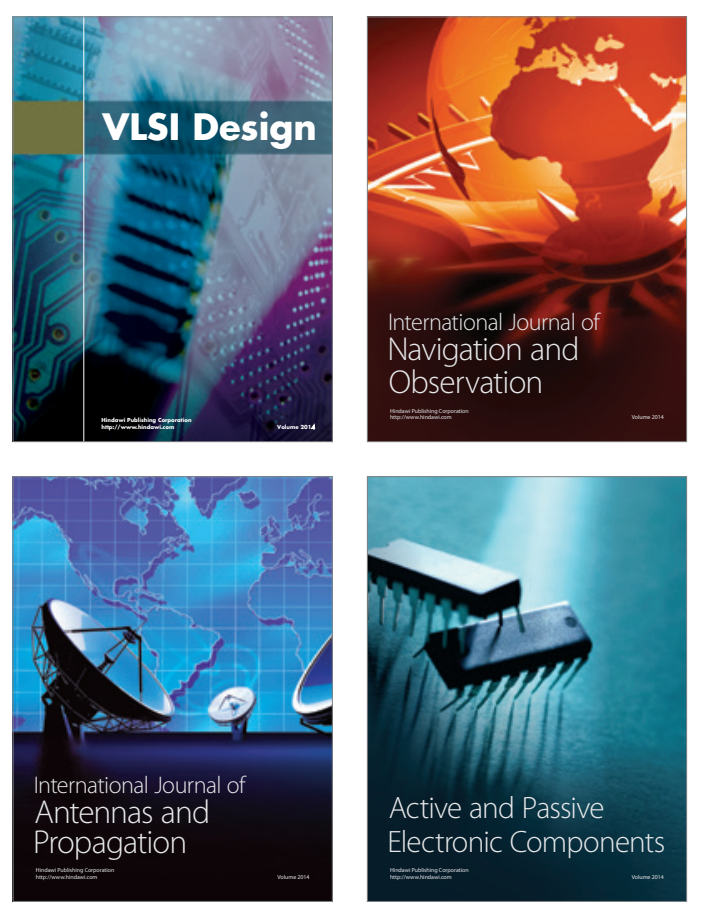
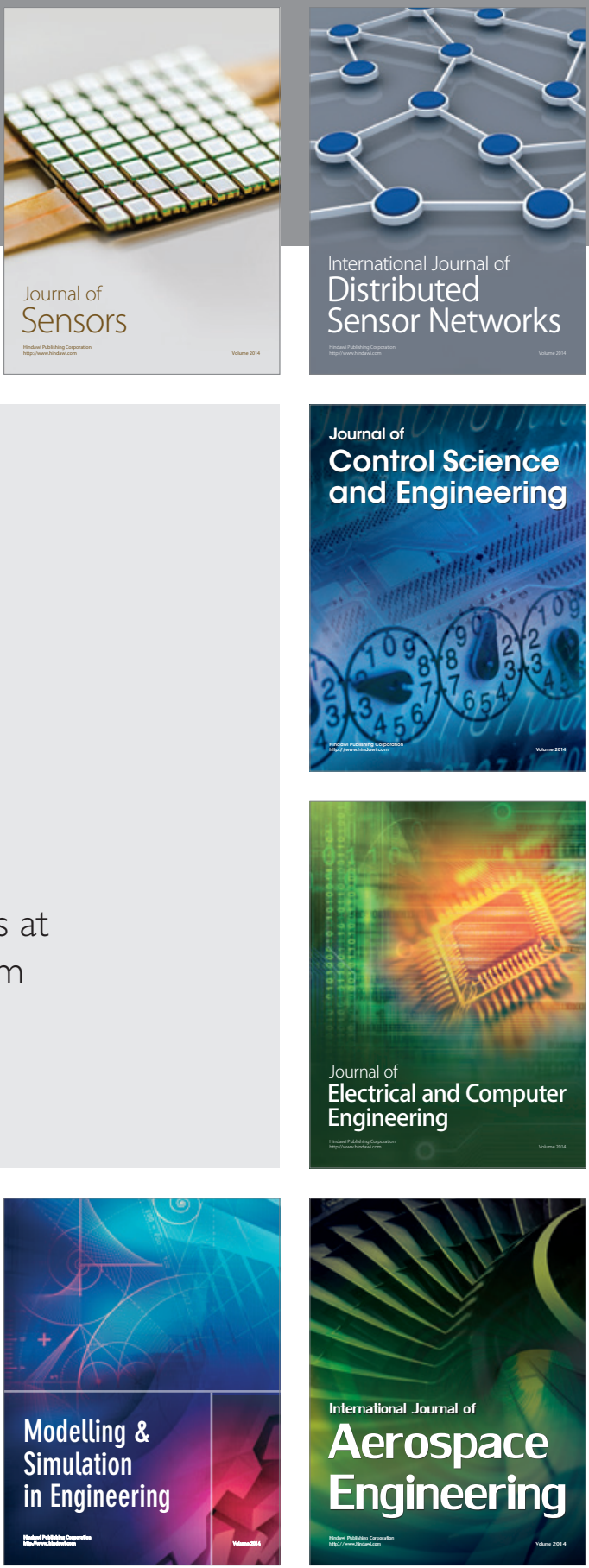

Journal of

Control Science

and Engineering
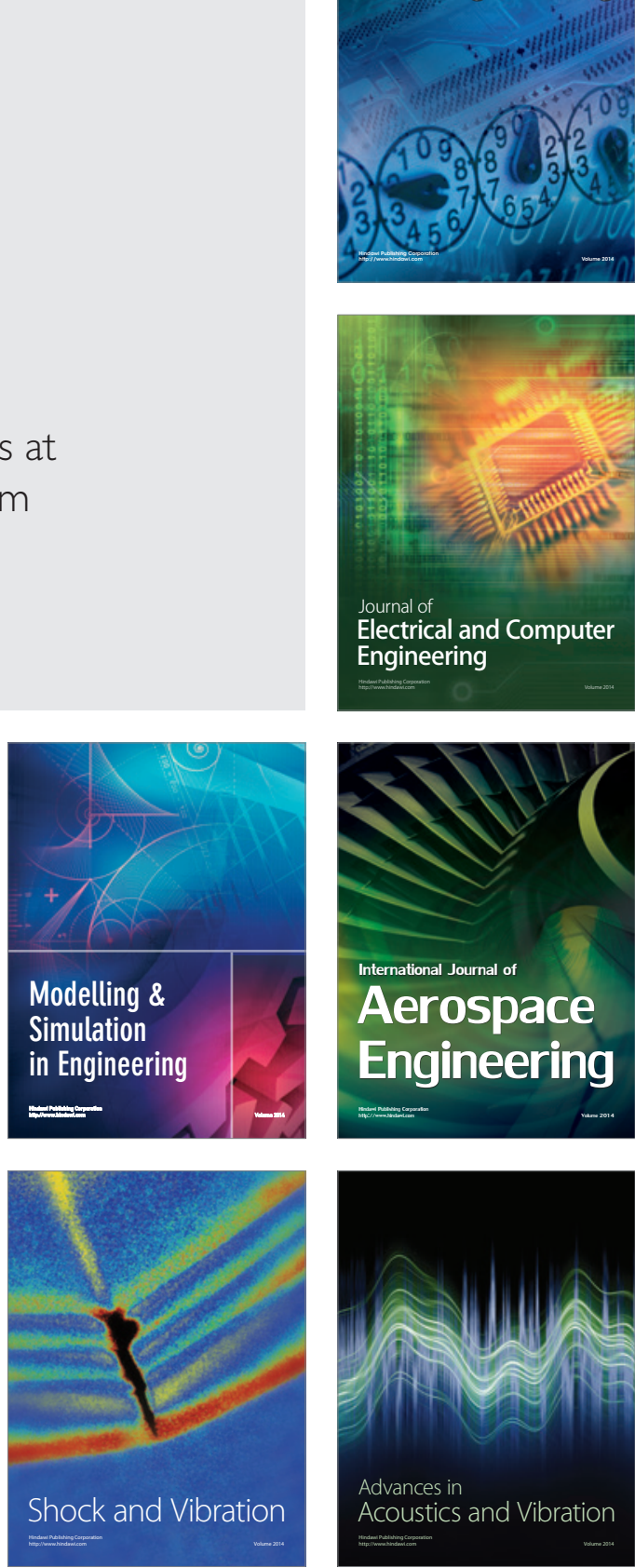